\title{
Atraumatic Ruptured Giant Renal Tumor
}

\author{
Gezahen Negusse Ayane, ${ }^{1}$ Khutsafalo Kadimo ${ }^{2}$
}

1. University of Botswana, Faculty of Medicine, Botswana

2. University of Botswana, Library, Botswana

Correspondence to: Gezahen Ayane, email:medpeter2003@yahoo.fr.

\section{Summary}

Massive hemoperitoneum due to spontaneous rupture of renal cell carcinoma is a rare presentation during emergency laparotomy. A 60-year-old female patient presented with severe abdominal pain, nausea, vomiting and abdominal distension. A massive hemoperitoneum secondary to ruptured left kidney tumor was found during laparotomy. Histology confirmed a papillary renal cell carcinoma. Ruptured renal cell carcinoma can be a cause of bleeding into the retroperitoneum space. Emergency sonography and laparotomy are advocated for its management.

\section{Introduction}

While post-traumatic retroperitoneal hemorrhage of renal cell carcinoma has previously been described, but cases of intraperitoneal atraumatic rupture of renal cell carcinoma are very few. Since Wunderlich first described spontaneous renal hemorrhage in 1856 , the literature has few reports about this uncommon case (1). In most cases, intraperitoneal or retroperitoneal renal rupture is secondary to trauma, and atraumatic renal rupture associated with a hemorrhage is associated with pre-existing diseases of the renal parenchyma such as vascular abnormality, infection, hydronephrosis, coagulopathy, and renal neoplasms (2). Literature also shows that $60 \%$ of spontaneous renal bleeding cases are associated with a renal tumor.

Renal neoplasms can be classified as benign or malignant. Benign renal neoplasm (papillary adenomas, renal oncocytomas, metanephric adenomas, and fibroadenomas) are not commonly cited in literature as causes of spontaneous intraperitoneal or intraperitoneal hemorrhage (1-3). Malignant renal neoplasms are divided as conventional "clear cell" renal cell carcinoma $70 \%$, papillary renal carcinoma $10-15 \%$, chromophobe renal carcinoma $5 \%$, collecting duct carcinoma $<1 \%$, and renal cell carcinoma 4-5\% "which is an unclassified category". The classical triad clinical presentation of renal cell carcinoma of flank pain, abdominal mass, and hematuria
Keywords: Renal cell carcinoma, Rupture, Complex mass, Nephrectomy, Papillary

Ann Afr Surg. 2020; 17(3):130-131

DOI: http://dx.doi.org/10.4314/aas.v17i3.9

Conflicts of Interest: None

Funding: None

(C) 2020 Author. This work is licensed under the Creative Commons Attribution 4.0 International License.

Submitted: 16 May 2019

Revised: 4 November 2019

Accepted: 10 December 2019

Online first: 29 May 2020

is uncommon and clinically, papillary renal cell carcinoma can have an atypical presentation, making this case an important contribution to existing literature.

\section{Case report}

A 60-year-old female patient presented with severe abdominal pain, nausea, vomiting and abdominal distension, which started about 12 hours prior to the examination. The patient denied any previous abdominal trauma or hematuria. At the time of examination, the patient appeared to be very sick looking but with a normal temperature. Her blood pressure and pulse were $95 / 50 \mathrm{mmHg}$ and 115 per minute. She was also noted to have a distended and tender abdomen. Baseline blood investigation showed hemoglobin $5.6 \mathrm{~g} / \mathrm{dL}$, slightly elevated urea, and creatinine. Emergency abdominal ultrasound revealed a massive intra-abdominal free fluid and a huge complex mass in the left side of the abdomen. The right kidney was normal while the left kidney was not visualized. After a short period of intensive resuscitation, the patient was taken for emergency exploratory laparotomy with a diagnosis of acute abdomen and intra-abdominal bleeding of unknown cause. Intraoperatively, a left radical nephrectomy was performed to manage a ruptured left kidney with massive hemoperitoneum (about $2.5 \mathrm{~L}$ ) and necrosis within and 
around it (Fig. 1). Histology report indicated a solid tumor, grey-white-brown appearance with marked

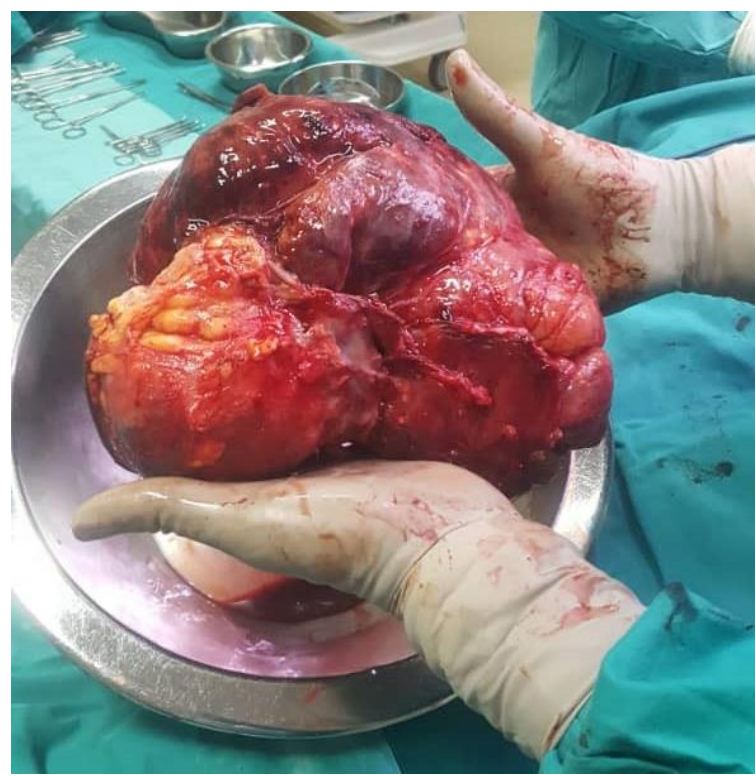

Figure 1. Giant renal tumor

necrosis and hemorrhage. The tumor measured $29 \times 20 \times 10$ $\mathrm{cm}$, weighed $5 \mathrm{~kg}$ and involved major calyces. However, the renal pelvis and the ureter were free of the tumor. During the postoperative period, the patient remained stable in the ward with normal vitals, normal urine output, and was discharged without local or systemic complications. Consequently, the patient had two followups in the surgical outpatient clinic (15 days after discharge from the surgical ward and 45 days after the first follow-up). The patient was then transferred to oncology for further management.

\section{Microscopy description}

This was a moderately differentiated papillary renal cell carcinoma with prominent hemorrhage and necrosis. The tumor involved the fibrotic pseudo capsule and small cluster tumor cells were also noted in the renal vein, but the perirenal fat tissue and the ureter were free from the tumor.

\section{Discussion}

Renal cell carcinoma represents one of the ten most common malignancies with predominance in male over female (1.5:1) (4). Despite the classical triad clinical presentation, most cases remain completely asymptomatic and non-palpable until the late stage of the disease. In our case, we do not have a clear patient history to confirm the above symptoms.

The most common cause of renal bleeding is secondary to trauma, and the spontaneous rupture and bleeding of renal cell carcinoma is rare. Renal cell carcinoma was first described by Wunderlich in 1856 (2). Renal parenchymal diseases such as vascular abnormality, infections, hydronephrosis, and renal tumor are predisposing factors for spontaneous rupture and bleeding. Most (60\%) cases of bleeding are related to renal malignancies (2). Among renal malignancies, angiomyolipomas followed by renal cell carcinoma are the most common causes of spontaneous rupture and bleeding (2). A sudden increase in intraparenchymal pressure due to a minimal effort like sneezing or coughing can result in spontaneous rupture and bleeding in cases of the causative malignant kidney (1).

In the absence of an abdominal CT scan, a bedside Focused Assessment with Sonography for Trauma (FAST) is the best modality investigation to assess unstable patients in the emergency department with spontaneous hemoperitoneum. Depending on the patient's hemodynamic status and the availability of other investigations, imaging can be used (4,5). An emergency midline laparotomy is also the best approach to deal with intra-abdominal bleeding and better control of the renal vascular pedicle. Surgery remains the only definitive approach for ruptured renal cell carcinoma, and indication for nephron-sparing surgery is well-known. Because of the poor prognosis of advanced renal cell carcinoma, post-surgery oncology follow-up is advised. However, chemotherapy as monotherapy is not useful; immunotherapy including tumor vaccine and a monoclonal antibody have proved effective $(4,6)$.

\section{Conclusion}

Spontaneous rupture and bleeding of renal cell carcinoma is a less common cause of hemoperitoneum. Urgent abdominal ultrasound can be used as a primary modality in the absence of CT scan. We recommend adequate preoperation resuscitation prior to emergency midline laparotomy.

\section{References}

1. Storkel S, Eble JN, Adlakha K, et al. Classification of renal cell carcinoma: Workgroup no. 1. Cancer. 1997;80:987-989.

2. Hora M, Hes O, Klečka J, et al. Rupture of papillary renal cell carcinoma. Scand J Urol Nephrol. 2004;38:481-484.

3. Skinner DG, Colvin RB, Vermillion CD, et al. Diagnosis and management of renal cell carcinoma A clinical and pathologic study of 309 cases. Cancer (0008543X). 1971;28:1165.

4. Ljungberg B, Cowan NC, Hanbury DC, et al. EAU Guidelines on Renal Cell Carcinoma: The 2010 Update. Eur Urol. 2010; 58:29-38.

5. Muneer A, Harrison SCW, Browning AJ. Atraumatic retroperitoneal haemorrhage in a hydronephrotic kidney secondary to transitional cell carcinoma. Int $\mathrm{J}$ Clin Pract. 2004;58:726-727.

6. Cohen HT, McGovern FJ. Renal cell carcinoma. N Engl J Med. 2005;353:2477-2490. 